\title{
TECHNICAL MODIFICATION OF THE NUSS OPERATION FOR THE CORRECTION OF PECTUS EXCAVATUM
}

José Ribas Milanez de Campos, Marcelo Heleno Fonseca, Eduardo de Campos Werebe, Manuel Carlos Prieto Velhote, and Fábio Biscegli Jatene

Pectus excavatum is a deformity of the thoracic wall present in less than $1 \%$ of the population. Treatment is essentially surgical because of the size of the deformity. The most employed surgical options are the technique proposed by Ravitch ${ }^{1}$ and its variations and the technique proposed by Nuss. ${ }^{2}$ The first one is considered the conventional technique, while the Nuss procedure is minimally invasive and is currently receiving wide acceptance.

The goal of this report is to propose 2 technical modifications to the Nuss operation regarding the position in which the patient is placed on the surgery table and the site of introduction of the optical trocar.

\section{DESCRIPTION OF THE PROPOSED TECHNIQUE}

The patient is placed in the supine position, under general anesthesia and orotracheal intubation. The patient lies on a $12 \mathrm{~cm}$-high cushion that extends from the head to the waist and is placed longitudinally on the surgical table, parallel to the median line (Figure 1). The patient's arms are stretched along the body and fixed to the surgical table. The optical trocar $\left(5 \mathrm{~mm}\right.$ and $\left.30^{\circ}\right)$ is inserted 2 intercostal spaces cranially with respect to the space where the metal bar will be introduced at the median axillary line.

Otherwise, the operative technique remains as originally described by Nuss. ${ }^{2}$

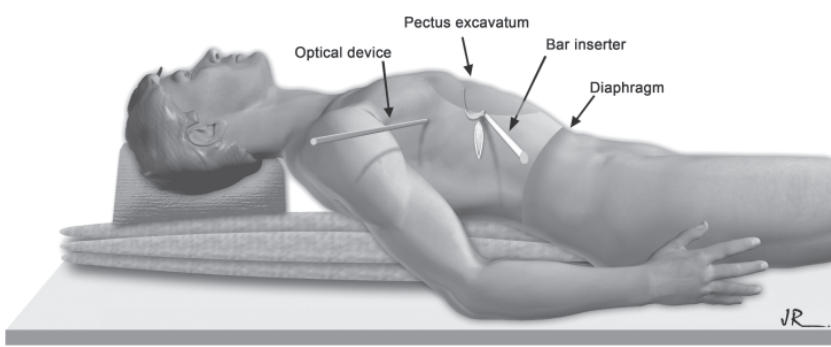

Figure 1

Thoracic Surgery Service, Hospital das Clínicas, Faculty of Medicine, University of São Paulo.

Email: jribas@usp.br

\section{COMMENTS}

In the technique originally described by Nuss, the arms of the patient are kept abducted at $90^{\circ}$ in relation to the body, and the the optical trocar is introduced 2 intercostal spaces caudal to the introduction point for the metal bar (Figure 2).

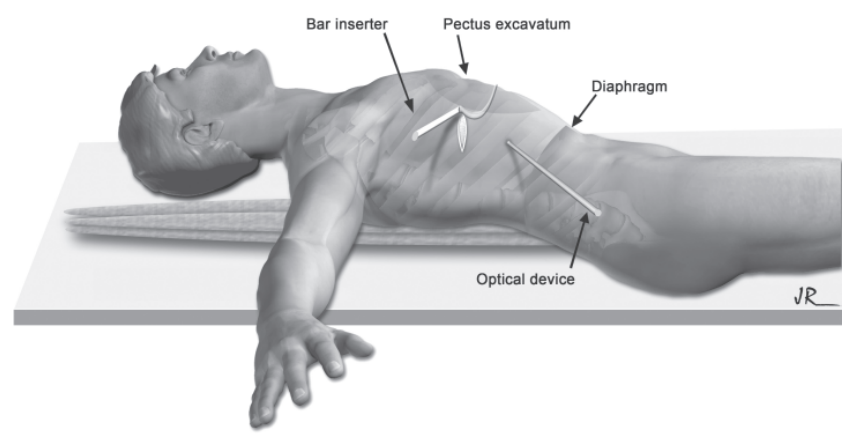

Figure 2

We propose a new technical approach in which we change the patient's arm position and introduce the optical trocar above the introduction point for the metal bar. The following benefits of this new approach have been observed as detailed below:

1. Increased safety regarding the introduction of the trocar; this is because this procedure is carried out at a higher level in the pleural cavity, thus reducing the risk of a diaphragmatic lesion.

2. Easier handling of the optical device; this enables better visualization of the bar inserter during dissection of the anterior mediastinum.

3. Optimization of the resources offered by the $30^{\circ}$ optical device, with better visualization due to the possibility of complete rotation; this enables working laterally or under the bar inserter.

4. Better visibility of the pleural cavity, of the sites to be handled, and of the dissected tunnel near the posterior 
wall of the sternum; thus, the pectus excavatum deformity does not obstruct optical image.

5. Easier identification of the intrathoracic vessels, such as the internal thoracic arteries, and a better view of the left pleural cavity in cases in which this cavity is open.

6. Easier passing of the fixation wires of the metal bar in the right hemithorax to avoid its displacement.

7. Better positioning of a thoracic drain, if necessary in case of aerial fistulae, using the trocar orifice; this position is closer to the apex of the thoracic cavity.

8. An aesthetically more acceptable result; the small scar, which is approximately $5 \mathrm{~mm}$, is covered by the patient's arms due to its location closer to the axillary cavity.

9. Last, but not least, minimization of the possibility of a brachial plexus lesion; this is due to the placement of the arms of the patient along his/her body.

We have employed these two technical modifications in 6 patients who underwent surgery in our Thoracic Surgery Department over the last 4 months, and we have observed the efficacy and the benefits of this technique. We thus consider it important to share this information because we are convinced that it can contribute to better performance of this surgery.

\section{REFERENCES}

1. Ravitch MM. The operative treatment of pectus excavatum. Ann Surg. $1949 ; 129: 429-44$.
2. Nuss D, Kelly RE, Croitoru DP, Katz ME. A 10-year review of a minimally invasive technique for the correction of pectus excavatum. $\mathrm{J}$ Pediatr Surg. 1998;33:545-52. 